\title{
Linkage and Antiretroviral Therapy Within 72 Hours at a Federally Qualified Health Center in New Orleans
}

\author{
Jason Halperin, MD, MPH, Isolde Butler, MD, MPH, Katherine Conner, MPH, \\ Leann Myers, PhD, , Pamela Holm, BA, Logan Bartram, MD, and Nicholas Van Sickels, MD ${ }^{1}$
}

W HILE HIV INCIDENCE declines nationally, the Southern United States remains disproportionally affected. New Orleans ranks third in the United States for the rate of new HIV infections. ${ }^{1}$ Previous studies in San Francisco, Haiti, and South Africa have shown that immediate initiation of antiretroviral therapy (ART) leads to earlier virologic suppression, increased retention in care, and decreased mortality. ${ }^{2-4}$ Further, the publication of three influential studies showed no linked transmission events in patients when ART results in viral suppression. ${ }^{5}$ Scientific advances demonstrate that effective treatment with ART prevents sexual HIV transmission. ${ }^{6}$ The Centers for Disease Control and Prevention has endorsed the concept of $\mathrm{U}=\mathrm{U}$ (undetectable $=$ untransmittable.) ${ }^{7}$

The Federally Qualified Health Center (FQHC), CrescentCare, partnered with the New Orleans Office of Health Policy, to start all newly diagnosed patients on ART within $72 \mathrm{~h}$ of diagnosis. The CrescentCare Start Initiative (CCSI) enhances navigation, expedites clinic intake, and initiates immediate ART. CCSI started on December 1, 2016, and in October 2017 underwent an analysis comparing the first 8 months to a matched cohort.

A Standard Operating Procedure (SOP) was disseminated agency wide, including training sessions for all providers. CCSI optimized provider availability and the navigator scheduled patients into any available slot. The intervention leveraged existing CrecentCare-run HIV testing sites, its sexually transmitted diseases (STD) clinic, and informed the clinic's referral network. A navigator was available $24 \mathrm{~h}$ per day to coordinate linkage of new diagnoses. The intake process for CCSI was streamlined for a focused HIV visit with a treating provider. Initial visit was $30 \mathrm{~min}$. The first dose of ART was directly observed. Providers were encouraged to use tenofovir alafenanide/entricitabine (TAF/FTC) + dolutegravir as the initial regimen. It was chosen for the regimen's effectiveness, tolerability, and empiric coverage of hepatitis B, and approved use in patients with a creatinine clearance greater than or equal to $30 \mathrm{~mL} / \mathrm{min}$. After the visit, baseline HIV labs were drawn, and patients saw case management, behavioral health, and eligibility specialists when indicated.
A medical checklist was developed to standardize workflow. All primary-care providers committed to seeing patients for the initial visit. Referral was then made to an HIV specialist for on-going management. Labs were reviewed within $48 \mathrm{~h}$. An HIV specialist regularly reviewed patient encounters. Follow-up appointments were made within 4 weeks and were longer in duration. The patient navigator assisted with all appointments through the second provider visit. The provider or patient could decline therapy through shared decision making.

In October, CCSI was compared to a cohort from the previous year. The comparator group tested positive at CrescentCare community testing sites and used the same navigator to minimize confounders. The 29 patients in the cohort saw an HIV specialist for their initial visit and were started on an integrase-based initial regimen (Table 1).

Viral suppression was defined as an HIV RNA less than 200 copies $/ \mathrm{mm}^{3}$. Time to suppression was defined as days from diagnosis to viral suppression. All patient information and data points were complete through October 6, 2017. For patients who transferred care, the last viral load was used for analysis. Continuous characteristics were compared using Wilcoxon's rank-sum test, given that normality could not be assumed. HIV viral load was log-transformed before analysis. Pearson's $\chi^{2}$ test was used to compare categorical variables (Fisher's exact test when sample size assumptions were not met). Time to suppression (median and 95\% confidence interval) was estimated using the Kaplan-Meier product-limit method; the logrank test was used to assess differences in time to suppression between the two groups of patients. Analyses were conducted using SAS software v. 9.4 (SAS Institute, Inc., Cary, NC).

Seventy-seven patients with a new diagnosis of HIV were referred to CCSI from December 5, 2016, to August 6, 2017. $92 \%(71 / 77)$ were linked, saw a treating provider, and started ART within $72 \mathrm{~h}$ of diagnosis. Four of the six patients not linked within $72 \mathrm{~h}$ were linked to care within 30 days of diagnosis. $69 \%$ (49/71) were seen within $24 \mathrm{~h}$ of diagnosis, and the remainder $(31 \%, 22 / 71)$ within $72 \mathrm{~h}$. Tenofovir

\footnotetext{
${ }^{1}$ CrescentCare, New Orleans, Louisiana.

${ }^{2}$ Global Biostatistics and Data Science, School of Public Health and Tropical Medicine, Tulane University New Orleans, New Orleans, Louisiana.

${ }^{3}$ Department of Internal Medicine, Tulane University School of Medicine, New Orleans, Louisiana

(C) Jason Halperin, et al., 2018; Published by Mary Ann Liebert, Inc. This Open Access article is distributed under the terms of the Creative Commons Attribution Noncommercial License (http://creativecommons.org/licenses/by-nc/4.0/) which permits any noncommercial use, distribution, and reproduction in any medium, provided the original author(s) and the source are credited.
} 
Table 1. Demographics

\begin{tabular}{lcc}
\hline & $\begin{array}{c}\text { CSI }(\mathrm{n}=71), \\
\mathrm{n}(\%)\end{array}$ & $\begin{array}{c}\text { Historical } \\
\text { cohort } \\
(\mathrm{n}=29), \mathrm{n}(\%)\end{array}$ \\
\hline Male & $51(71.8)$ & $27(93.1)$ \\
Transgender & $2(2.8)$ & $2(6.9)$ \\
African American & $38(52.8)$ & $19(65.5)$ \\
Latino & $7(9.9)$ & $2(6.9)$ \\
MSM & $39(54.9)$ & $24(82.8)$ \\
Heterosexual & $28(39.4)$ & $5(17.2)$ \\
People who inject & $3(4.2)$ & $7(24.1)$ \\
$\quad$ drugs & $38(53.5)$ & $18(62.1)$ \\
Uninsured at & $15(21.1)$ & $8(27.6)$ \\
$\quad$ baseline & $22(31)$ & $11(37.9)$ \\
Syphilis & $4(5.7)$ & $0(0)$ \\
GC/CT & $5(7.3)$ & $1(3.5)$ \\
Hep C & $24(34.3)$ & $15(51.7)$ \\
Homeless & $16(22.4)$ & $6(20.7)$ \\
Tobacco use & & \\
Drug use other & $31.1+10.5$ & $32.3+10.4$ \\
than THC & 419 cells/mm & $408 \mathrm{cells} / \mathrm{mm}{ }^{3}$ \\
Age (mean + SD) & & \\
Baseline CD4 & & \\
$\quad$ count (median) & $41,900 \mathrm{copies} / \mathrm{mL}^{3}$ & $36,130 \mathrm{copies} / \mathrm{mL}$ \\
Baseline viral & & \\
$\quad$ load (median) & & \\
\hline
\end{tabular}

There were significant differences between gender, sexual orientation, and intravenous drug use between the two groups.

CCSI, CrescentCare Start Initiative; GC/CT, gonorrhea/chlamydia; MSM, men who have sex with men; THC, marijuana.

alafenamide/emtricitabine + dolutegravir was started in 69 patients. Two patients received an alternate regimen, one with end-stage renal disease and another who seroconverted on PrEP. All 71 patients were started on ART and had their first dose directly observed.

CCSI was compared to a cohort of 29 patients diagnosed and linked between December 2015 and August 2016. The mean time to linkage in the historical cohort was 30 days (95\% CI: $25.1-43.6$ days) compared to 1.3 days (95\% CI: $1.09-1.51$ days $)$ in CCSI $(p<0.0001)$. The median time to viral suppression $\left(<200\right.$ copies $/ \mathrm{mm}^{3}$ ) in the historical cohort was 68 days (95\% CI: 60-92 days) compared to 30 days (95\% CI: $27-34$ days $)$ in CCSI $(p<0.0001)$. There were no significant differences between the groups by baseline viral load, CD4 count, or ART regimen (Fig. 1).

$23 \%(14 / 61)$ of CCSI patients had transmitted resistance. Two had NRTI resistance with the M184V/I mutation and 12 had NNRTI resistance. All patients with transmitted resistance achieved viral suppression. Ten genotypes failed or were not performed at baseline. All 71 CCSI patients achieved viral suppression. By the end of the study period, viral suppression was maintained in 70 of 71 patients [median 135 days, interquartile range $(\mathrm{IQR})=117$; Q1 $=90, \mathrm{Q} 3=207]$; one patient had rebound viremia due to nonadherence.

CrescentCare has witnessed a significant increase in referrals to the clinic following implementation. At the time of article drafting, 112 patients have accessed immediate treatment. The successes of this intervention include high rates of linkage to care, earlier viral suppression, and sustained impact over time. A full-time navigation specialist was essential for patient engagement. The use of an SOP and medical checklist ensured that non-HIV specialists could provide this model of care. This model is dependent on sameday accessibility. The FQHC structure with guaranteed sameday appointments, extended hours, and wrap-around services lends itself very well to an immediate start initiative.

Previous studies have shown poorer health outcomes when HIV care is provided by clinicians with lower HIV caseloads. ${ }^{8}$ This intervention, due to the requirement of immediate accessibility, incorporated a potential first appointment with a generalist to initiate ART and then referral to a specialist. Patients were seen, triaged, and treated effectively, but further analysis of long-term outcomes is necessary.

The limitations of this study are that it was nonrandomized and the control used for analysis was historical. There were significant differences between the two groups, including gender, sexual orientation, and intravenous drug use (IVDU). Of these, IVDU has been shown to decrease rates of viral suppression ${ }^{9}$ and could have impacted this comparison. Further scaling up of this intervention will require targeted resources to invest in patient navigation, immediate

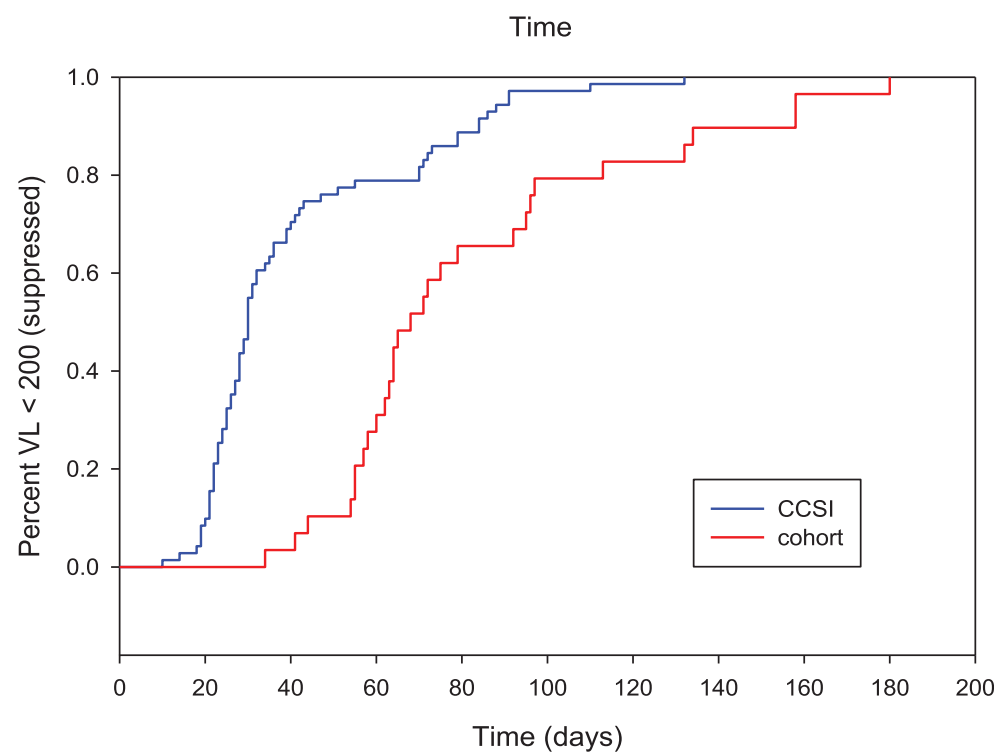

FIG. 1. The relationship between $\%$ virally suppressed $(<200$ copies $/ \mathrm{mL})$ and time to viral suppression among patients newly diagnosed with HIV infection as part of the CCSI compared to historical cohort. This Kaplan-Meier plot shows the proportion of patients with viral load $<200$ copies/mL HIV RNA over time. Time to viral suppression for patients in the CrescentCare Start Initiative was a median of 30 days (blue line) and significantly shorter than for patients treated in the historical cohort for 68 days (red line). CCSI, CrescentCare Start Initiative. (Color image can be found at www.liebertonline.com/ apc). 
patient access, and coverage for the cost of the first 30 days of medication.

The success of this intervention depends on its sustainability, future retention-in-care outcomes, and continued viral suppression. To reach the 2020 United Nations goal of $90 / 90 / 90,{ }^{10}$ the impact must be sustained. The HIV epidemic in the Southern United States requires urgent and novel interventions. Rapid access to therapy improves health outcomes and renders our patients uninfectious. Immediate initiation of ART can be effectively provided in a community FQHC and play a significant role in ending the HIV epidemic.

\section{Acknowledgments}

Dr. L.M. was supported, in part, by U54 GM104940 from the National Institute of General Medical Sciences of the National Institutes of Health, which funds the Louisiana Clinical and Translational Science Center.

\section{Author Disclosure Statement}

No competing financial interests exist.

\section{References}

1. Singh S, Song R, Johnson A, et al. (CDC). HIV Incidence, Prevalence, and Undiagnosed Infections in Men Who Have Sex with Men. Presented at: Conference on Retroviruses and Opportunistic Infections, 2017, Seattle, WA.

2. Pilcher CD, Ospina-Norvell C, Dasgupta A, et al. The effect of same-day observed initiation of antiretroviral therapy on HIV viral load and treatment outcomes in a US Public Health Setting. J Acquir Immune Defic Syndr 2017; 74:44-51.
3. Koenig SP, Dorvil N, De vieux JG, et al. Same-day HIV testing with initiation of antiretroviral therapy versus standard care for persons living with HIV: A randomized unblinded trial. PLoS Med 2017;14:e1002357.

4. Rosen S, Maskew M, Fox MP, et al. Initiating antiretroviral therapy for HIV at a patient's first clinic visit: The RapIT randomized controlled trial. PLoS Med 2016;13:e1002015.

5. Rodger AJ, Cambiano V, Bruun $\mathrm{T}$, et al. Sexual activity without condoms and risk of HIV transmission in serodifferent couples when the HIV-positive partner is using suppressive antiretroviral therapy. JAMA 2016;316:171-181.

6. $\mathrm{U}=\mathrm{U}$ taking off in 2017. Lancet HIV 2017;4:e475.

7. McCray E, Mermin J. Dear colleague: September 27, 2017. 2017. Available at: www.cdc.gov/hiv/library/dcl/dcl/092717. html (Last accessed November 5, 2017).

8. Landovitz RJ, Desmond KA, Gildner JL. Quality of care for HIV/AIDS and for primary prevention by HIV specialists and nonspecialists. AIDS Patient Care STDS 2016; 30:395-408.

9. Westergaard RP, Hess T, Astemborski J, et al. Longitudinal changes in engagement in care and viral suppression for HIVinfected injection drug users. AIDS 2013;27:2559-2566.

10. Corless IB, Hoyt AJ, Tyer-Viola, et al. 90-90-90-Plus: Maintaining adherence to antiretroviral therapies. AIDS Patient Care STDS 2017;31:227-236.

Address correspondence to: Jason Halperin, MD, MPH

CrescentCare

2601 Tulane Avenue, 5th Floor New Orleans, LA 70119

E-mail: jason.halperin@crescentcare.org 